\title{
Use of an open-source CAD software program and additive manufacturing technology to design and fabricate a definitive cast for retrofitting a crown to an existing removable partial denture
}

Fang-Yu Su DDS ${ }^{1}$, Jen-Chih Tsai DDS ${ }^{2}$, Dean Morton BDS ${ }^{3}$, MS Wei-Shao Lin DDS $^{4}$

1 Resident, Postdoctoral Program in Prosthodontics, Department of

Prosthodontics, Indiana University School of Dentistry, Indianapolis, Ind

2 Private practice, Hsinchu City, Taiwan, Republic of China

3 Professor and Chair, Department of Prosthodontics, Indiana University School of Dentistry, Indianapolis, Ind

4 Associate Professor, Department of Prosthodontics, Indiana University School of Dentistry, Indianapolis, Ind

This is the author's manuscript of the article published in final edited form as:

Su, F.-Y., Tsai, J.-C., Morton, D., \& Lin, W.-S. (2019). Use of an open-source CAD software program and additive manufacturing technology to design and fabricate a definitive cast for retrofitting a crown to an existing removable partial denture. The Journal of Prosthetic Dentistry, 122(4), 351-354. https://doi.org/10.1016/j.prosdent.2019.02.017 


\begin{abstract}
This technical report describes a digital process for designing and fabricating a stackable definitive cast and die system to facilitate the fabrication of a new surveyed crown to retrofit to a removable partial denture (RPD). By using an open-source computer-aided design (CAD) software program, this technique provides an economical option for dental clinicians and laboratory technicians to use intraoral scans and design a stackable definitive cast and die system with minimal financial investment in the CAD software. In addition, this technique provides the advantage of a conventional indirect technique in that it can create a definitive cast with an RPD clasp assembly ready for the dental technician to properly contour the new surveyed crown, but without the need for the patient to be without the RPD during the process.
\end{abstract}

Retrofitting a new surveyed crown to an existing removable partial denture (RPD) is indicated when the existing RPD is clinically acceptable and the abutment tooth is 
clinically sound. 1 It is considered one of the most labor-intensive chairside and laboratory procedures. 2 Killebrew3 described a direct technique in 1961, in which an acrylic resin or wax pattern was fabricated directly on the prepared abutment tooth intraorally and then cast. Barrett and Pilling4 presented an indirect technique in 1965, in which the definitive impression for a new surveyed crown was made with the existing RPD in situ. A definitive cast was then created in the dental laboratory, with the RPD available for the dental technician to properly contour the new surveyed crown. The direct technique requires increased chair time to create the proper contour of the surveyed crown, and the indirect technique often requires the patient to be without the RPD while a new surveyed crown is fabricated in the dental laboratory.2, 3, 4

The rapid development of the computer-aided design and computer-aided manufacturing (CAD-CAM) technology has transformed dentistry in the past decades. 5 Several clinical reports have described the use of proprietary CAD software programs to design a new surveyed crown fitting an existing RPD.6, 7, 8, 9 These proprietary CAD software programs can be costly; however, using an open-source software program without the financial investment has been described in various clinical indications.10, 11 The purpose of this report was to describe an alternative technique for designing a stackable definitive cast and die system to facilitate the fabrication of a new surveyed crown to retrofit an existing RPD.

Technique 
1. Use appropriate soft-tissue management around the abutment tooth for the new surveyed crown and make an intraoral scan with the RPD in situ by using an intraoral scanner (TRIOS 3; 3Shape A/S) (Fig. 1). Export the file in standard tessellation language (STL) format.

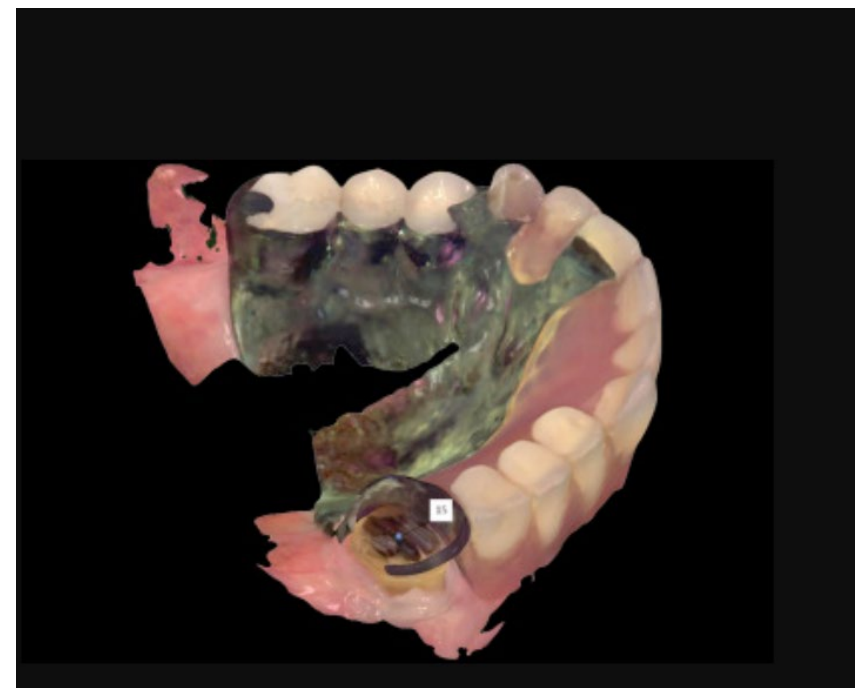

Figure 1. Intraoral scan including abutment for new surveyed crown and removable partial denture (RPD) in situ.

2. Import the STL file into an open-source CAD software program (MeshMixer v11.0.544; Autodesk). Remove the excess area on the intraoral scans by using the "Select" function and hotkey " $X$ " to delete the selected area.

3. Use the "Select" function to specify the desired area for removable dies for the abutment tooth and clasp assembly (Fig. 2A, B). Separate the selected area for the removable dies from the virtual cast by using the "Separate" function (Meshmixer, v11.0.544; Autodesk) (Fig. 2C). 


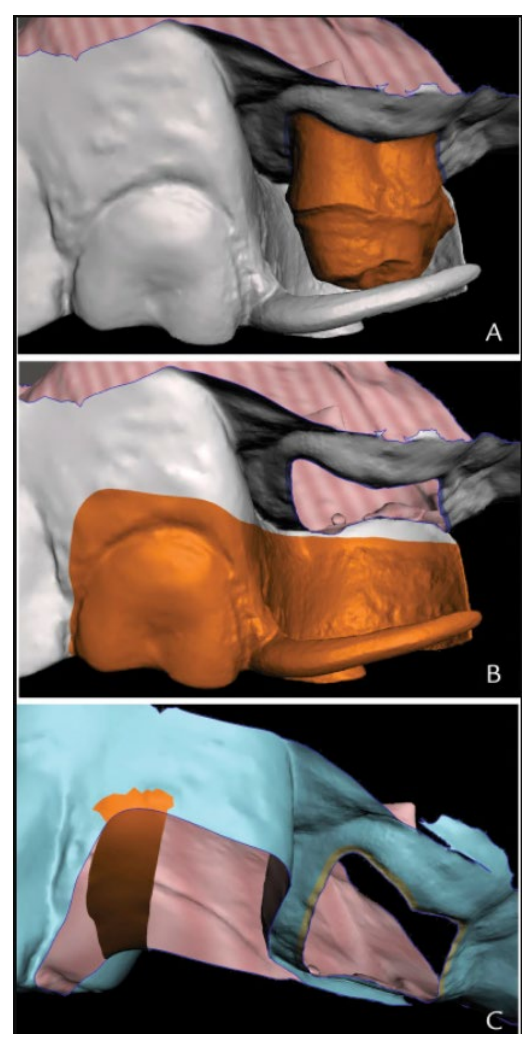

Figure 2. A, Separate abutment tooth from definitive virtual cast. B, Separate clasp assembly from definitive virtual cast. C, Desired area for removable dies of abutment tooth and clasp assembly separated from definitive virtual cast.

4. After separating the removable dies, fill the empty space on the virtual cast and removable dies by using the "Inspector" function in the "Analysis" tab and/or the "Bridge" function in the "Select" tab. Make the maxillary and mandibular casts solid with the "Make solid" function in "Edit."

5. Select the removable die of the abutment tooth for the surveyed crown and extrude as flat-end extrusion to form a post (Fig. 3A). Turn on the "Show objects browser" function from the "View" tab in the top menu bar. Duplicate the removable die from the "Objects browser." Select the duplicated die and click the "Offset" function to create a $0.08-\mathrm{mm}$ offset around the entire abutment and post (Fig. 3B). After the 
offset is formed on the duplicate die, select the virtual cast and then select the duplicate die; use the "Boolean difference" function in the "Edit" tab to create post

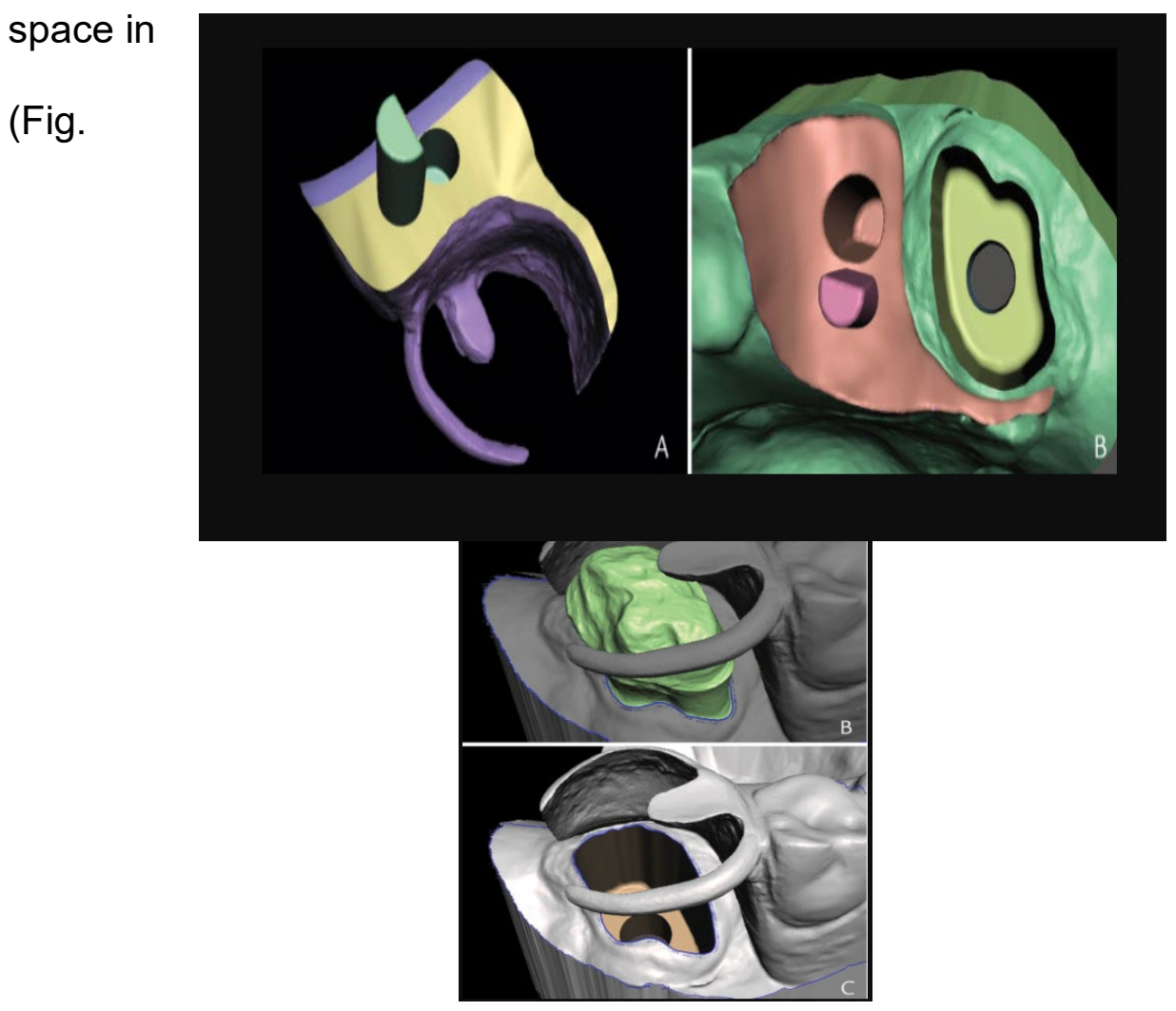
the virtual cast $3 C)$.

Figure 3. A, Flat-end extrusion completed on removable die of abutment tooth to form post. B, Duplicated removable die with $0.08-\mathrm{mm}$ circumferential offset in situ with virtual definitive cast. C, Post space created in virtual definitive cast with "Boolean difference" function.

6. Create the retentive pin for the removable die of the clasp assembly by using "Cylindrical object" in the "Meshmix" function (Fig. 4A). Orient the retentive pin following the direction of the guide plane from the existing RPD. Form a removable die with the clasp assembly and post space by following the same procedures as steps 4 and 5 (Fig. 4B) 
Figure 4. A, Removable die of clasp assembly with retentive pins created. B, Post space created in virtual definitive cast with "Boolean difference" function.

7. Export the STL files of the definitive cast and removable die of the abutment tooth. Send these STL files to a desktop stereolithography (SLA) 3D printer (Asiga MAX; Asiga) and fabricate the objects by using compatible model resin (VeriModel OS Ivory; Whip Mix Corp) (Fig. 5A, B). Send the STL file of the removable die of the clasp assembly to a direct metal printing (DMP) 3D printer (ProX DMP 200; 3D Systems) to manufacture the object in cobalt-chromium (Co-Cr) alloy (LaserForm $\mathrm{CoCr}(\mathrm{C}) ; 3 \mathrm{D}$ Systems) (Fig. 5C). Assemble the printed definitive cast and printed removable die of the abutment tooth and clasp assembly together to ensure the proper fit of all components (Fig. 5D). 


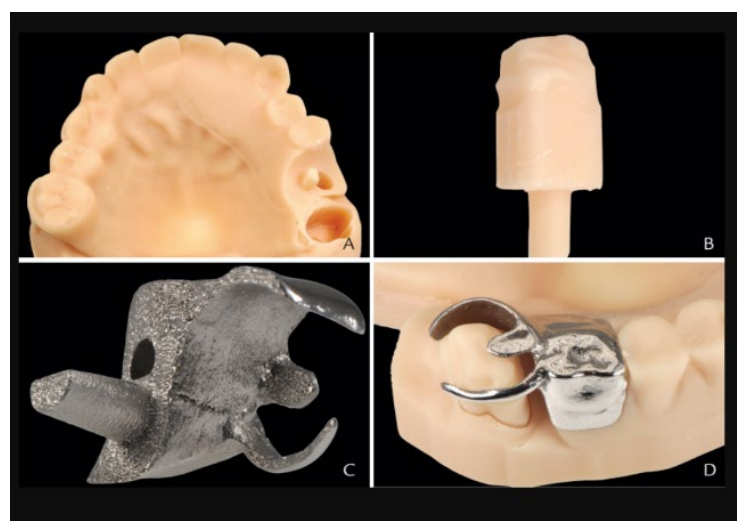

Figure 5. A, Printed definitive cast. B, Printed removable die of abutment tooth. C, Printed cobalt-chromium (Co-Cr) removable die of clasp assembly. D, Removable dies properly fit into definitive cast.

8. Fabricate the surveyed crown either by the conventional lost-wax casting technique or CAD-CAM process, providing the surveyed crown with passive contacts with the components of the clasp assembly when the removable dies are fully seated on the cast.

\section{DISCUSSION}

The use of an open-source CAD software program is gaining popularity in digital dentistry. The described technique explored the possibilities of using an open-source CAD software program to facilitate an alternative clinical solution for designing a stackable definitive cast and die system to be used for the fabrication a new surveyed crown to retrofit an existing RPD. The most significant benefits of using an open-source CAD software program are it does not require the expense of software installation and there is no fee associated with exporting the design files. This minimal financial investment is within the reach of most dentists and laboratory technicians. The software is capable of selectively removing, 
merging, or superimposing multiple digital files based on the user's needs. The described technique mirrors the conventional indirect technique as the intraoral scan was made with the existing RPD in situ. However, it offers the additional benefit that a definitive cast with an RPD clasp assembly can be created in the dental laboratory for the dental technician to properly contour the new surveyed crown fitting the RPD, without the need for the patient to be without RPD during the process.

The limitations of using this technique include the initial learning curve for the dental clinicians and laboratory technicians in using an open-source CAD software program with no predefined dental workflow established in the software. Navigating and developing a different dental workflow could be time-consuming and challenging for new software users.

\section{SUMMARY}

This technical report describes a digital process by using an open-source CAD software program and additive manufacturing technology to design and fabricate a stackable definitive cast and die system to facilitate the fabrication of a new surveyed crown to retrofit an RPD.

\section{REFERENCES}

1. Smith, C. S., Grandin, H., \& Low, K. (2019). A predictable all-digital workflow to retrofit a crown to an existing removable prosthesis. The Journal of Prosthetic Dentistry, 121(6), 876-878. https://doi.org/10.1016/j.prosdent.2018.08.014 
2. Tran, C. D., Sherraden, D. R., \& Curtis, T. A. (1986). A review of techniques of crown fabrication for existing removable partial dentures. The Journal of Prosthetic Dentistry, 55(6), 671-673. https://doi.org/10.1016/0022-3913(86)90439-7

3. Killebrew, R. H. (1961). Crown construction for broken down partial denture abutments. The Journal of Prosthetic Dentistry, 11(1), 93-94. https://doi.org/10.1016/0022-3913(61)90115-9

4. Barrett, D. A., \& Pilling, L. O. (1965). The restoration of carious clasp-bearing teeth. The Journal of Prosthetic Dentistry, 15(2), 309-311. https://doi.org/10.1016/0022$\underline{3913(65) 90102-2}$

5. Alghazzawi, T. F. (2016). Advancements in CAD/CAM technology: Options for practical implementation. Journal of Prosthodontic Research, 60(2), 72-84. https://doi.org/10.1016/j.jpor.2016.01.003

6. Marchack, B. W., Chen, L. B., Marchack, C. B., \& Futatsuki, Y. (2007). Fabrication of an all-ceramic abutment crown under an existing removable partial denture using CAD/CAM technology. The Journal of Prosthetic Dentistry, 98(6), 478-482. https://doi.org/10.1016/S0022-3913(07)60148-6

7. Yoon, T.-H., \& Chang, W.-G. (2012). The fabrication of a CAD/CAM ceramic crown to fit an existing partial removable dental prosthesis: A clinical report. The Journal of Prosthetic Dentistry, 108(3), 143-146. https://doi.org/10.1016/S0022$\underline{3913(12) 60137-1}$

8. Kerdani, T. E., \& Roushdy, S. (2017). The Use of CAD/CAM Technology for Fabricating Cast Gold Survey Crowns under Existing Partial Removable Dental 
Prosthesis. A Clinical Report. Journal of Prosthodontics, 26(4), 321-326.

https://doi.org/10.1111/jopr.12525

9. Lee, J.-H. (2016). Completely digital approach to fabricating a crown under an existing partial removable dental prosthesis by using an intraoral digital scanner in a single appointment. The Journal of Prosthetic Dentistry, 115(6), 668-671. https://doi.org/10.1016/j.prosdent.2015.11.021

10.Piedra Cascón, W., \& Revilla-León, M. (2018). Digital workflow for the design and additively manufacture of a splinted framework and custom tray for the impression of multiple implants: A dental technique. The Journal of Prosthetic Dentistry, 120(6), 805-811. https://doi.org/10.1016/j.prosdent.2018.02.003

11. McAllister, P., Watson, M., \& Burke, E. (2018). A Cost-Effective, In-House, Positioning and Cutting Guide System for Orthognathic Surgery. Journal of Maxillofacial and Oral Surgery, 17(1), 112-114. https://doi.org/10.1007/s12663-017$\underline{1067-y}$ 